\section{BLUETOOTH COMMUNICATION FOR BATTERY POWERED MEDICAL DEVICES}

\author{
Branko Babušiak - Štefan Borik
}

\begin{abstract}
- wireless communication eliminates obtrusive cables associated with wearable sensors and considerably increases patient comfort during measurement and collection of medical data. Wireless communication is very popular in recent years and plays a significant role in telemedicine and homecare applications. Bluetooth technology is one of the most commonly used wireless communication types in medicine. This paper describes the design of a universal wireless communication device with excellent price/performance ratio. The said device is based on the low-cost RN4020 Bluetooth module with Microchip Low-energy Data Profile (MLDP) and due to low-power consumption is especially suitable for the transmission of biological signals (ECG, EMG, PPG, etc.) from wearable medical/personal health devices. A unique USB dongle adaptor was developed for wireless communication via UART interface and power consumption was evaluated under various conditions.
\end{abstract}

Keywords: bluetooth, wireless communication, low energy, medical device

\section{INTRODUCTION}

Wireless communication is increasingly popular in medicine. Wearable medical devices such as heart rate monitors or glucose meters with wireless communication allow immediate data transfer and considerably increase patient comfort during measurements. Wireless communication has an irreplaceable place in telemedicine. It enables health care assistance of individuals from virtually anywhere by means of modern communication protocols [1]. Bluetooth technology represents one type of wireless communication protocol used in medicine. Bluetooth Low Energy (BLE), marketed as Bluetooth Smart, is aimed at novel applications in healthcare, fitness and home entertainment applications. Compared with previous Bluetooth generations, BLE has similar communication range but offers considerably reduced power consumption and costs [2-4]. According to Bluetooth SIG (Special Interest Group), energy consumption improvements in BLE result in devices that can operate for "months or years" on a single button cell. Furthermore, BLE offers smaller footprint, lowered cost and wide compatibility with smart phones, tables and computers.

Our design is based on the Bluetooth low energy module manufactured by Microchip. We developed a Bluetooth receiver-transmitter pair consisting of two identical modules, the first acting as a central role (PC) and the second one in peripheral role (medical sensor). We then conducted a series of experiments to evaluate the power effectiveness of the constructed devices and also tested the communication range in a standard environment.

\section{MOTIVATION AND MODULE CHOICE}

We design and construct various medical device prototypes at our department. Some of them are wearable, requiring battery powered operation and wireless data transfer. Thus, power consumption is a crucial parameter in the design process. Our primary goal was to design a universal wireless communication device pair with the best price/performance ratio. While there are many Bluetooth modules available in the market, we focused solely on low-cost, low energy units compatible with Bluetooth version 4.1. The said version provides data transfer speed of up to $24 \mathrm{Mbit} / \mathrm{s}$. Figure 1 shows our survey of select low-energy modules available on the market, including the power consumption and module price. The current consumption values were taken from manufacturer datasheets of respective modules and represent active data transmit/receive conditions.

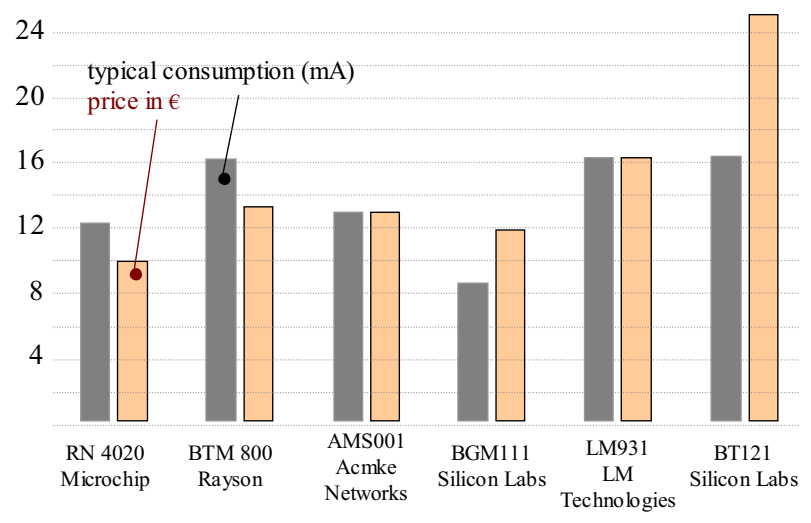

Fig. 1. Current consumption and price of select low-energy Bluetooth ver.4.1 modules

Real-life current consumption values of each module may vary because of different conditions (data rate, distance, etc.) during measurements. While the RN4020 module does not offer the lowest current consumption, it compensates this with the lowest price (i 10 EUR) from the reviewed modules. The RN4020 by Microchip is fullycertified Bluetooth version 4.1 module and general specifications and electrical characteristics are listed in Table $1[5]$.

\footnotetext{
* Department of Electromagnetic and Biomedical Engineering, University of Žilina, Žilina, Slovakia branko.babusiak@fel.uniza.sk, stefan.borik@fel.uniza.sk
} 
Table 1. Specifications and characteristics of RN4020

\begin{tabular}{c|c}
\hline Specification & Description \\
\hline Standard & Bluetooth 4.1 \\
Frequency Band & $2.42 .48 \mathrm{GHz}$ \\
Maximum Data Rate & $1 \mathrm{Mbps}$ \\
Operation Range & 100 meters \\
Interface & UART, PIO, AIO, SPI \\
RF TX Power & $+7 \mathrm{dBm}($ average $)$ \\
Encryption & AES128 \\
Operating temperature & $-30{ }^{\circ} \mathrm{C}$ to $+85^{\circ} \mathrm{C}$ \\
Supply Voltage & $1.8-3.6 \mathrm{~V} \mathrm{DC}$ \\
Working current & $12 \mathrm{~mA}($ typical $)$ \\
Standby current & $<0.5 \mathrm{~mA}$ \\
Idle & $<1.5 \mathrm{~mA}$ \\
Deep Sleep & $<5.0 \mu \mathrm{A}$ \\
Dormant & $<700 \mathrm{nA}$ \\
\hline
\end{tabular}

Table 2. Average current consumption (with MCU) at $3 \mathrm{~V}$

\begin{tabular}{c|c}
\hline Configuration & Current consumption \\
\hline MCU only & $2.6 \mathrm{~mA}$ \\
Deep Sleep & $3.3 \mathrm{~mA}$ \\
Active (discoverable) & $5.5 \mathrm{~mA}$ \\
Connected & $5.9 \mathrm{~mA}$ \\
Sending Data $\left(f_{s}=500 \mathrm{~Hz}\right)$ & $7.6 \mathrm{~mA}$ \\
Sending Data $\left(f_{s}=1000 \mathrm{~Hz}\right)$ & $7.9 \mathrm{~mA}$ \\
\hline
\end{tabular}

Power consumption measurements of the RN4020 module were performed in order to verify datasheet values since power consumption is a crucial parameter in battery powered medical devices. The average current consumption at input voltage of $3 \mathrm{~V}$ is shown in Table 2. The first row shows the current consumption of the MCU with Bluetooth module powered off. The MCU is represented by the ATmega328p manufactured by Atmel. Subsequent rows show the current consumption of the module in various scenarios, including the influence of two different sampling frequencies. Higher sample rates slightly increase the power consumption, although the manufacturer-declared typical current consumption was never exceeded.

\section{COMMUNICATION SET}

Our design uses MLDP for wireless communication, offering low energy consumption and sufficient data rate for transferring basic medical signals. Unfortunately, MLDP is not natively supported by Bluetooth 4.1 as a profile and thus two RN4020 modules are required for communication via MLDP.

\subsection{USB dongle - Central Role module}

The first module is configured in central role mode. The said mode allows for the following functions:

- scan for nearby peripheral modules;

- establishment of connection with a particular module;

- data transfer function;
- connection termination.

The central Bluetooth module is to be connected to PCs, notebooks or smartphones through standard USB port. Received Bluetooth data can be read through the UART interface. The UART interface is also used for module control via simple ASCII commands and to read output status messages from the module itself. The popular FT232RL integrated circuit is used as USB to serial UART interface convertor. The FT232RL driver is supported under Windows, Linux and Mac operating systems. The central mode hardware prototype is similar to a USB dongle.

No external power supply is necessary as the dongle draws power from the USB port. The module is powered by the $+3.3 \mathrm{~V}$ output from the internal low dropout (LDO) regulator of the FT232RL. It is possible to draw up to $50 \mathrm{~mA}$ current from the regulator which is sufficient for stable module operation (see Table I). The hardware design of the USB dongle is very simple and consists of the RN4020 module, the FT232RL converter and three indicator LEDs. The hardware design does not include the controlling microcontroller unit (MCU).

LED1 (green) indicates that the module is awake and active; LED2 (red) indicates MLDP data is received and LED3 (green) indicates the module is connected to a peripheral device. Pins CTS and RTS need to be interconnected between the Bluetooth module and FT232 because of hardware handshaking which prevents data loss. Pin CMD/MLDP is used to enter either Command or MLDP mode. In Command mode, UART traffic is sent to a command interpreter. In MLDP mode, UART traffic is routed to the MLDP Bluetooth. The module is woken up from deep sleep by pulling pin WAKE_SW high. In the designed USB dongle this pin is permanently pulling high and thus the module is always active.

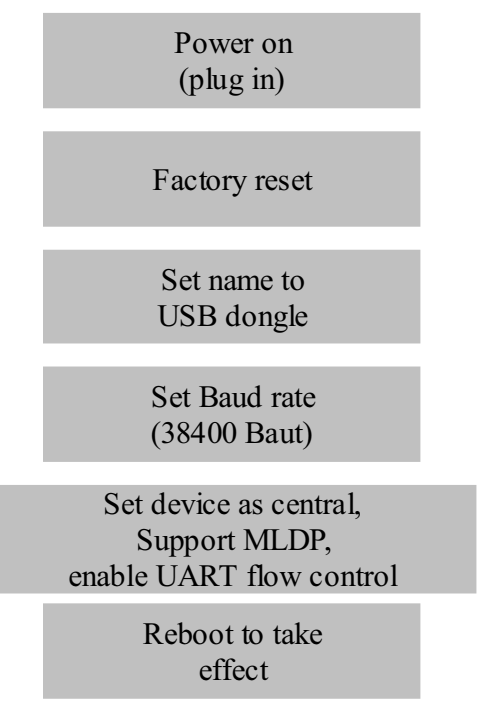

Fig. 2. Flowchart of module in central role 


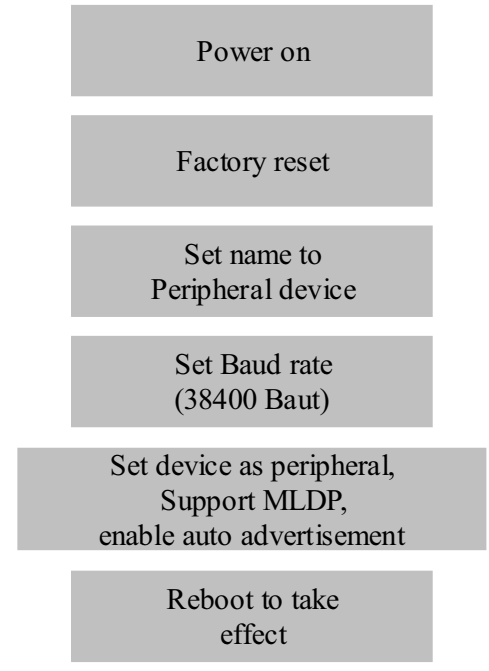

Fig. 3. Flowchart of module in peripheral role

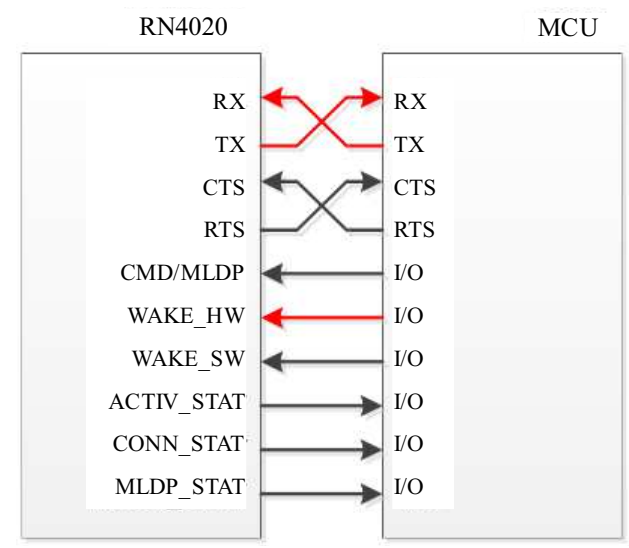

Fig. 4. Peripheral module and MCU interconnection, [5]

When the USB dongle is plugged into a USB port it enters the Command mode. The device name, roles, Bluetooth services, UART Baud rate and many other features can be set in this mode. Configured features are stored in permanent memory and are loaded after a restart or when the USB dongle is plugged in again. The Bluetooth module included in the USB dongle is configured according to the flowchart shown in Fig. 2. This is the default configuration for the USB dongle.

\subsection{Medical Device Module in Peripheral Role}

Power dissipation was the most important parameter in the peripheral module design and evaluation process. Basic interconnection between the peripheral module and the MCU is depicted on diagram in Fig. 4. The MCU can be directly replaced by any type of medical sensor with UART interface since only three wires (red color in Fig. 4) are necessary for communication purposes. CTS and RTS will remain unconnected if there are no hardware handshaking pins. If the module is configured according to the flowchart in Fig. 3, the CMD/MLDP pin should permanently hold high for MLDP mode. The module goes to dormant mode or wake up procedure from the said mode by changing the logic level on WAKE_HW pin. Outputs ACTIV_STAT, CONN_STAT and MLDP_STAT are indicating the following states: active, connected and MLDP data receive, respectively. These outputs can be connected directly to indicating LEDs instead of the MCU inputs.

\subsection{Interconnecting the Two Modules}

Let us assume that both modules are configured according to the previous flowcharts. When the USB dongle is plugged in and the peripheral module is powered on, the connection will be automatically established. The central module is scanning for peripheral devices on first connection attempt to get the MAC address of the device. Once the MAC address is determined or known beforehand, scanning is not necessary during the subsequent poweron phases. Connection is established by ASCII command from the central module using the MAC address of the peripheral device.

After the peripheral module is connected to the central module, it is switched from the command mode to MLDP mode by pulling DTR line of UART low. The module will output MLDP to indicate the start of the MLDP mode. From now on all UART traffic is routed to the MLDP Bluetooth. If DTR pin is pulled high, the module will switch back to the Command mode.

\section{COMMUNICATION SOFTWARE}

Accompanying software was designed in C\# programming language to test the modules. It allows viewing of one-channel signal in online mode. The software creates ASCII commands for the USB dongle and controls all communication.

The sawtooth signal is generated by the MCU which is connected with module RN4020 using only two wires RX and TX. The WAKE_SW pin is connected to $+3.3 \mathrm{~V}$ so the module is permanently in wake up state and no hardware handshaking is used. The MCU generates sawtooth samples at a sample rate of $1 \mathrm{kHz}$ and starts generating samples when it receives the $\mathrm{S}$ character and stops generating samples when the $\mathrm{K}$ character is received.

The artificial sawtooth signal can be replaced by the MCUs analog-to-digital converter input signal, enabling the transmission and display of any analog signals, including medical signals such as ECG, EMG, EOG or EEG.

\section{AREAS OF APPLICATION}

The designed communication set was successfully implemented and tested in two medicine-related projects. The first project is aimed at online monitoring of ECG. The Bluetooth module is integrated into the ECG central unit.

The electrodes are connected to the unit through microUSB connectors with power supply pins, enabling the 


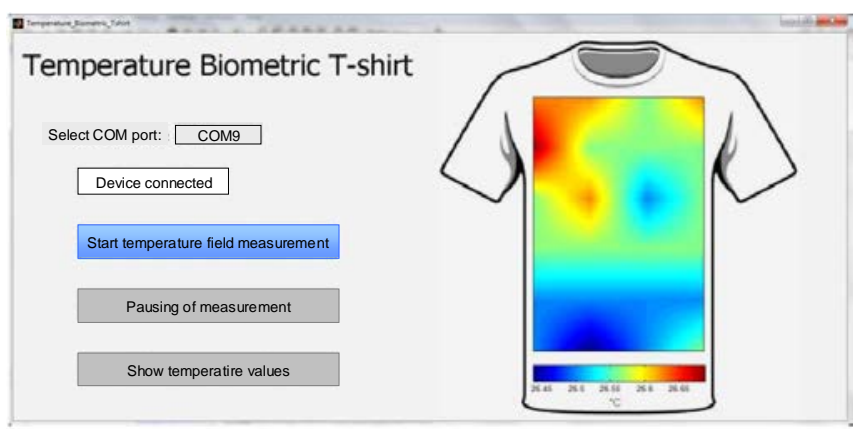

Fig. 5. Application for monitoring the chest temperature
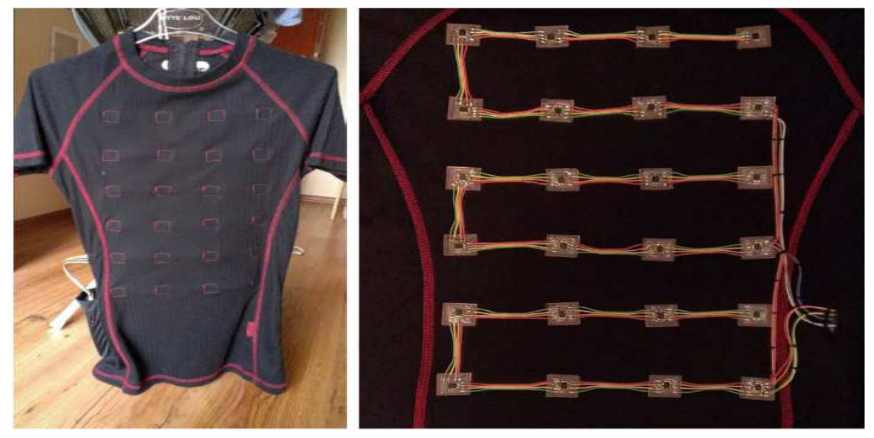

Fig. 6. Biometric T-shirt and inside sensors detail, right

[3] REICHERT, S.-GASS, R.-HAJJAM, A.-KOUKAM, A.ANDRES, E.: An Optimized Communication for Emergency Health Situations, eHealth, Telemedicine, and Social Medicine, 2009. eTELEMED '09. International Conference, 31-35, (2009).

[4] DAE-KICHO-SEUNG-HOONLEE-CHANG, A.-MASSEY, T.-CHIA-WEICHANG-MIN-HSIEHTSAI- SARRAFZADEH, M.-GERLA, M. : Opportunistic medical monitoring using bluetooth P2P networks, World of Wireless, Mobile and Multimedia Networks, (2008).

[5] (2015, February). RN4020, RN4020 datasheet. [Online]. Available: http://ww1.microchip.com/downloads/en/Device Doc/50002279A.pdf.

[6] BABUSIAK, B.-GALA, M.-PENHAKER, M.-CERNY, M.-KRAUS, J.: Dry contact less surface electrodes for bioelectrical measurements, IEEE 12th International Symposium on Applied Machine Intelligence and Informatics (SAMI) (2014), 357-360.

[7] BABUSIAK, B.-BORIK, S.: Bio-Amplifier with programmable gain and adjustable leads, Telecommunications and Signal Processing (TSP), 2013 36th International Conference (2013), 616-619.

[8] BABUSIAK, B.-GALA, M.-BARABAS, B.: Design of One-lead ECG Data Logger, Telecommunications and Signal Processing (TSP), 2014 37th International Conference, 518-52.

Received 8 October 2015 munication unit connected to a PC or a notebook. It acts as a virtual serial port and enables easy implementation of communication in a broad range of software. Moreover, the Microchip Low-energy Data Profile (MLDP) can be used in Android devices with Bluetooth 4.0 and the signal can be directly displayed on smart phone or tablet devices.

\section{Acknowledgment}

This work was supported by the Slovak Research and Development Agency under the contract No APVV-140519 .

\section{REFERENCES}

[1] CERNY, M. :PENHAKER, M. : Wireless body sensor network in Health Maintenance systems, Elektronika ir Elektrotechnika, (9), 113-116, (2011).

[2] YOUNG-JINPARK-HUI-SUPCHO : Transmission of ECG data with the patch-type ECG sensor system using Bluetooth Low Energy, ICT Convergence (ICTC), 2013 International Conference, 289- 294, (2013).
Branko Babušiak was born on 17 January 1983. In 2006 he graduated (MSc) at the Department of Electromagnetic and Biomedical Engineering of the Faculty of Electrical Engineering at University of Žilina. He defended his $\mathrm{PhD}$ in the field of technical cybernetics in 2009 at the Department of Measurement and Control of the Faculty of Electrical Engineering and Computer Science at VSB-Technical University of Ostrava; his thesis title was Detection of eye artefacts in EEG record. Since 2009 he is working as a tutor with the Department of Electromagnetic and Biomedical Engineering in Žilina. His scientific research is focusing on digital signal processing of biological signals and biomedical image processing.

Štefan Borik was born in 1986. He received his MSc degree in 2011 and PhD degree in 2014 at Faculty of Electrical Engineering, Department of Electromagnetic and Biomedical Engineering, University of Žilina. Currently, he is working as assistant professor at Department of Electromagnetic and Biomedical Engineering, University of Žilina. He especially deals with analog and digital hardware design, biological signal processing and modelling. 